\title{
The putative lytic transglycosylase VirB1 from Brucella suis interacts with the type IV secretion system core components VirB8, VirB9 and VirB11 \\ Correspondence \\ Christian Baron \\ baronc@mcmaster.ca \\ Received 6 July 2005 \\ Revised 16 August 2005 \\ Accepted 30 August 2005 \\ Christoph Höppner, ${ }^{1}$ Anna Carle, ${ }^{1}$ Durga Sivanesan, ${ }^{2}$ Sabine Hoeppner ${ }^{3}$ and Christian Baron ${ }^{1,2}$

\author{
${ }^{1}$ Ludwig-Maximilians-Universität, Department Biologie I, Bereich Mikrobiologie, Maria-Ward-Str. \\ 1a, D-80638 München, Germany \\ ${ }^{2}$ McMaster University, Department of Biology, 1280 Main St West, Hamilton, ON, Canada \\ LS8 4 K1 \\ ${ }^{3}$ Ludwig-Maximilians-Universität, Gene Center, Feodor-Lynen Str. 25, D-81377 München, \\ Germany
} \\ VirB1-like proteins are believed to act as lytic transglycosylases, which facilitate the assembly of type IV secretion systems via localized lysis of the peptidoglycan. This paper presents the biochemical analysis of interactions of purified Brucella suis VirB1 with core components of the type IV secretion system. Genes encoding VirB1, VirB8, VirB9, VirB10 and VirB11 were cloned into expression vectors; the affinity-tagged proteins were purified from Escherichia coli, and analyses by gel filtration chromatography showed that they form monomers or homo-multimers. Analysis of protein-protein interactions by affinity precipitation revealed that VirB1 bound to VirB9 and VirB11. The results of bicistron expression experiments followed by gel filtration further supported the VirB1-VirB9 interaction. Peptide array mapping identified regions of VirB1 that interact with VirB8, VirB9 and VirB11 and underscored the importance of the C-terminus, especially for the VirB1-VirB9 interaction. The binding sites were localized on a structure model of VirB1, suggesting that different portions of VirB1 may interact with other VirB proteins during assembly of the type IV secretion machinery.
}

\section{INTRODUCTION}

Type IV secretion systems (T4SS) are used by many Gramnegative bacteria to translocate virulence factors into eukaryotic cells or to mediate conjugative transfer of broad-hostrange plasmids (Cascales \& Christie, 2003; Celli \& Gorvel, 2004; Llosa \& O'Callaghan, 2004; Zupan et al., 2000). T4SS are crucial determinants of host-pathogen interactions, which enable bacterial survival in widely different habitats, such as the rhizosphere of plants (Agrobacterium tumefaciens) and intracellular compartments of mammalian cells (Brucella species). The best-studied model is A. tumefaciens; its T4SS comprises 12 components, VirB1-VirB11 and VirD4. Biochemical, genetic and cell biological experiments suggest that VirB2-VirB11 constitute a membrane-spanning pore, which connects the inner and the outer membrane through periplasmic interactions and homo-oligomer formation, and an extracellular pilus (Cascales \& Christie, 2003; Christie, 2004). VirD4 links this channel to translocated substrates (Atmakuri et al., 2003; Kumar \& Das, 2002). VirB6, VirB7, VirB8, VirB9 and VirB10 constitute the core of the T4SS and

Abbreviations: S2B, Strepll buffer; T4SS, type IV secretion system(s). the substrate transfer route, although direct evidence for channel formation is still lacking. VirB2 and VirB5 are major and minor components of the extracellular pilus of $A$. tumefaciens, which probably mediates contact formation with the host cell (Eisenbrandt et al., 1999; Hwang \& Gelvin, 2004; Schmidt-Eisenlohr et al., 1999). The role of the VirB3 protein is less well defined than those of other T4SS components, but its interaction with pilus components and localization in the outer membrane suggest that it may play a role during the assembly of this structure (Jones et al., 1994; Shamaei-Tousi et al., 2004; Yuan et al., 2005). VirB4 and VirB11 are multimeric inner-membrane-localized NTPases, which may traverse the inner membrane to contact periplasmic VirB proteins (Atmakuri et al., 2004; Dang \& Christie, 1997; Middleton et al., 2005; Yeo et al., 2003; Yuan et al., 2005). They may either act as assembly factors for the T4SS or drive pilus subunits or substrate molecules across the cell envelope. VirB2-VirB11 are indispensable both for gene transfer from A. tumefaciens and for Brucella's ability to reach the proper intracellular niche and to replicate within HeLa cells or macrophages (Berger \& Christie, 1994; Comerci et al., 2001; O'Callaghan et al., 1999; Sieira et al., 2000). 
VirB1 is the only non-essential T4SS component. It was previously demonstrated that VirB1 homologues play an important role in the T4SS of A. tumefaciens and Escherichia coli strains harbouring plasmids pKM101 and R1. The efficiency of substrate transfer was reduced 10- to 1000-fold upon non-polar deletion of the encoding genes (Bayer et al., 1995; Berger \& Christie, 1994; Fullner, 1998; Winans \& Walker, 1985). An infection assay with signature-tagged Brucella abortus mutants demonstrated that mutagenesis of the virB1 gene causes attenuation of virulence (Hong et al., 2000). A more recent study demonstrated that survival of $B$. abortus in macrophage cell cultures was attenuated in strains carrying a non-polar virB1 mutation (den Hartigh et al., 2004). Thus the deletion of genes encoding VirB1 homologues generally has an attenuating effect on T4SS-related functions. The Helicobacter pylori VirB1 homologue HP0523 is an exception to this rule, as it was shown to be essential for bacterial virulence (Odenbreit et al., 2001; Rohde et al., 2003). Due to the presence of highly conserved sequence motifs, VirB1 was identified as a putative lytic transglycosylase, but its specific role for T4SS function was not elucidated in detail. A. tumefaciens and Brucella suis VirB1 both possess a signal sequence and are therefore directed to the periplasmic space by the general secretion pathway (Llosa et al., 2000; O'Callaghan et al., 1999). Their enzymic activity probably leads to localized cell wall lysis, creating space for accommodation of the T4SS (Bayer et al., 2001; Mushegian et al., 1996; Zahrl et al., 2005). Despite the well-known area of lysozyme biochemistry, proposals for the catalytic mechanism of lytic transglycosylases were published only recently. Whereas the well-known lysozymes like hen egg white lysozyme break up murein by hydrolysis of the $\beta(1 \rightarrow 4)$-glycosidic bond between the $N$-acetylmuramic acid (MurNAc)-C1 and the $\mathrm{N}$-acetylglucosamine (GlcNAc)-C4, the lytic transglycosylases lyse this substrate in a transglycosylation reaction utilizing the $\mathrm{C} 6-\mathrm{OH}$ residue of the same MurNAc (Blackburn \& Clarke, 2001; Koraimann, 2003). Thus, no water is required for the reaction, which produces a 1,6-anhydromuramic acid terminal residue. Special features of the active site that distinguish the lytic transglycosylases from lysozymes must explain the mechanistic difference, and this question is subject to structural biological studies (Lehnherr et al., 1998; Leung et al., 2001; Mushegian et al., 1996; Thunnissen et al., 1994; van Asselt et al., 1999, 2000).

When VirB1 was identified as a lytic transglycosylase, its importance for the A. tumefaciens T4SS was largely attributed to its proposed catalytic activity. This notion was repeatedly confirmed by the observation that active-site mutants of the protein failed to fully complement virB1 deletion strains (Höppner et al., 2004; Mushegian et al., 1996). After export across the inner membrane, VirB1 of $A$. tumefaciens is further processed in the periplasm, yielding a processing product of the C-terminal 73 amino acids designated VirB1* (Baron et al., 1997). VirB1* and the $\mathrm{N}$-terminus, representing the lytic transglycosylase domain, independently enhanced tumorigenicity, which implied an additional function of VirB1* (Llosa et al., 2000). Further evidence for this hypothesis was generated when it was shown by co-immunoprecipitation that VirB9 interacts with VirB1* in A. tumefaciens (Baron et al., 1997). A highresolution dihybrid screen with protein components of the A. tumefaciens T4SS suggested self-interaction and a number of uni- and bidirectional interactions between VirB1 and VirB4, VirB8, VirB9, VirB10 and VirB11 (Ward et al., 2002). Direct biochemical evidence for the interactions was not presented in that study.

In spite of the low overall amino acid sequence identity of $22 \%$, the VirB1 homologue from B. suis complemented virB1 gene defects in A. tumefaciens, suggesting that it engages in similar interactions with T4SS components. Similarly, the B. suis VirB4 homologue complemented virB4 gene defects in A. tumefaciens (Yuan et al., 2005), which further supported the notion that the overall architecture of different T4SS is very similar (Christie, 2004; Yeo \& Waksman, 2004). To directly test interactions of VirB1sp with T4SS core components we chose derivatives of VirB proteins from B. suis, (abbreviated VirBs in the following or VirBsp to indicate periplasmic domains without signal peptides or membrane domains), which are more readily amenable to overproduction and purification than those from A. tumefaciens. Using different biochemical methods (affinity precipitation, gel filtration, bicistron expression, peptide array analysis), we showed that purified B. suis T4SS core components undergo different interactions. VirB1sp was found to interact with VirB9sp, and whereas this interaction was the strongest among those we investigated, VirB1sp also bound to VirB8sp and VirB11s. The binding sites were localized in a structure model of VirB1sp, suggesting that a sequence of transient interactions guides lytic transglycosylase function during T4SS assembly.

\section{METHODS}

Cultivation of bacteria. For overnight cultures all E. coli strains were grown in LB ( $1 \%$ tryptone; $0.5 \%$ yeast extract; $0.5 \% \mathrm{NaCl}$ ) or LBON ( $1 \%$ tryptone; $0.5 \%$ yeast extract) under aerobic conditions at $37^{\circ} \mathrm{C}$ in a laboratory shaker (modell Kühner, B. Braun) at 200 r.p.m. Carbenicillin $\left(100 \mu \mathrm{g} \mathrm{ml}^{-1}\right)$ was included for selection of plasmid-carrying cells. Day cultures were inoculated to an $\mathrm{OD}_{600}$ of 0.05 in vessels of appropriate volume with the same media under vigorous shaking at $37^{\circ} \mathrm{C}$ (Certomat-R, B. Braun Biotech International). The T7 promoter in the protein-overproducing strain GJ1158 (Bhandari \& Gowrishankar, 1997) was induced at an $\mathrm{OD}_{600}$ of $0 \cdot 4-0.8$ by addition of $5 \mathrm{M} \mathrm{NaCl}$ stock solution to a final concentration of $0.3 \mathrm{M}$. Cultivation under aerobic conditions then proceeded at different temperatures for varying amounts of time for the overproduction of specific proteins as follows: VirB1s (C- or Nterminal tag) at $26^{\circ} \mathrm{C}$ for $4 \mathrm{~h}$, VirB1s/VirBsX (bicistron constructs) at $26^{\circ} \mathrm{C}$ for $6 \mathrm{~h}$, VirB7sp at $26^{\circ} \mathrm{C}$ for $4 \mathrm{~h}$, VirB8sp at $37^{\circ} \mathrm{C}$ for $4 \mathrm{~h}$, VirB9sp at $37^{\circ} \mathrm{C}$ for $4 \mathrm{~h}, \operatorname{VirB10sp}$ at $27^{\circ} \mathrm{C}$ for $6 \mathrm{~h}$, VirB11s at $27^{\circ} \mathrm{C}$ for $18 \mathrm{~h}$. The total culture volumes were 1 litre, in four $500 \mathrm{ml}$ Erlenmeyer flasks each containing $250 \mathrm{ml} \mathrm{LBON}$.

Molecular biology methods. Manipulations of DNA for plasmid isolation, PCR amplification, restriction, ligation and sequencing followed standard procedures, using enzymes from New England Biolabs and MBI Fermentas and E. coli JM109 as cloning host (Maniatis et al., 1982; Yanisch-Perron et al., 1985). PCR fragments 
were first cloned into pCR2.1-TOPO (Invitrogen), followed by sequencing and further subcloning into expression vectors as described below.

Construction of virB gene expression vectors. Expression vectors for the production of VirBs proteins (Table 1) were constructed by PCR amplification of the genes with oligonucleotides, which introduced $5^{\prime}$ and $3^{\prime}$ restriction sites (sequences given in Table 2), followed by ligation into similarly cleaved vectors. Constructs for the overproduction of N-terminally tagged StrepIIVirB1sp and StrepIIVirB11s were generated by PCR amplification of the genes, cleavage with Acc65I/PstI and Acc65I/HindIII, and ligation into similarly cleaved pT7-7StrepII (pT7-7StrepIIVirB8sp, pT7-7StrepIIVirB9sp and pT7-7StrepIIVirB10sp were described previously; Yuan et al., 2005). The gene encoding VirB11s was subsequently subcloned using the same restriction sites into $\mathrm{pT} 7-\mathrm{H}_{6}$ TrxFus for expression as an N-terminally $\mathrm{His}_{6} \mathrm{TrxA}$-tagged fusion protein (pT7- $\mathrm{H}_{6}$ TrxVirB8sp, pT7- $\mathrm{H}_{6}$ TrxVirB9sp and pT7- $\mathrm{H}_{6}$ TrxVirB10sp were described previously; Yuan et al., 2005). Similar procedures were applied for the cloning of VirB7s into pT7- $\mathrm{H}_{6}$ TrxFus using XbaI/PstI restriction sites. pET24dVirB1spHis 6 for overproduction of C-terminally $\mathrm{His}_{6}{ }^{-}$ tagged VirB1sp was constructed by PCR cloning of the virB1 gene and introduction of the NcoI/NotI-treated fragment into similarly cleaved pET24d.

Bicistron vectors for the co-expression of proteins and vectors for the production of untagged control proteins were constructed as follows. In order to co-produce $\mathrm{C}$-terminally $\mathrm{His}_{6}$-tagged VirB1sp with putative interaction partners, vector pET21BC was constructed by introducing a SalI/NotI DNA fragment encoding Schizosaccharomyces pombe Srb11 including the ribosome-binding site (Baumli et al., 2005). The first ORF of the pET21BC series encoded the putative interaction partner and was created by cleavage of the vector with $\mathrm{NheI} / E \mathrm{coRI}$ and insertion of PCR-amplified virB8, virB9 and virB10 genes treated with the same restriction endonucleases. After excision of the Srb11-encoding gene by cleaving the vector with $\mathrm{NcoI} / \mathrm{NotI}$, virB1 was inserted using the same

Table 1. Plasmids

\begin{tabular}{|c|c|c|}
\hline Plasmid & Genotype & Source or reference \\
\hline pUC18VirB & Carb $^{\mathrm{R}}$, virB region from B. suis 1330 & O'Callaghan et al. (1999) \\
\hline pCR2 $2 \cdot 1-\mathrm{TOPO}$ & $\operatorname{Carb}^{\mathrm{R}} \mathrm{Kan}^{\mathrm{R}}$, for direct cloning of PCR fragments & Invitrogen \\
\hline pET24d & $\mathrm{Kan}^{\mathrm{R}}$, cloning and $\mathrm{T} 7$ expression vector & Novagen \\
\hline pT7-7StrepII & $\begin{array}{l}\text { Carb }^{\mathrm{R}} \text {, for overexpression of N-terminally StrepII-tagged fusion } \\
\text { proteins }\end{array}$ & Balsinger et al. (2004) \\
\hline pT7-7StrepIIVirB8sp & pT7-7StrepII with $492 \mathrm{bp}$ Acc65I/PstI fragment of virB8 from B. suis & Yuan et al. (2005) \\
\hline pT7-7StrepIIVirB9sp & pT7-7StrepII with $813 \mathrm{bp}$ Acc65I/PstI fragment of virB9 from B. suis & Yuan et al. (2005) \\
\hline pT7-7StrepIIVirB10sp & pT7-7StrepII with $1020 \mathrm{bp}$ Acc65I/PstI fragment of virB10 from B. suis & Yuan et al. (2005) \\
\hline pT77-StrepIIVirB11s & $\begin{array}{l}\text { pT7-7StrepII with } 1083 \mathrm{bp} \text { Acc65I/HindIII fragment of virB11 from } \\
\text { B. suis }\end{array}$ & This work \\
\hline pT7- ${ }_{6}$ TrxFus & $\begin{array}{l}\mathrm{Carb}^{\mathrm{R}} \text {, for T7-based expression of N-terminal } \mathrm{His}_{6} \text {-TrxA } \\
\text { (thioredoxin) fusions }\end{array}$ & Kromayer et al. (1996) \\
\hline pT7- ${ }_{6}$ TrxVirB7sp & pT7- ${ }_{6}$ TrxFus with 126 bp XbaI/PstI fragment of B. suis virB7 & This work \\
\hline pT7- ${ }_{6}$ TrxVirB8sp & pT7- $\mathrm{H}_{6}$ TrxFus with $492 \mathrm{bp}$ Acc65I/PstI fragment of B. suis virB8 & Yuan et al. (2005) \\
\hline pT7- ${ }_{6}$ TrxVirB9sp & pT7- $\mathrm{H}_{6}$ TrxFus with $813 \mathrm{bp}$ Acc65I/Pst fragment of B. suis virB9 & Yuan et al. (2005) \\
\hline pT7- $\mathrm{H}_{6} \operatorname{TrxVirB10sp}$ & pT7- $\mathrm{H}_{6}$ TrxFus with $1020 \mathrm{bp}$ Acc65I/PstI fragment of B. suis virB10 & Yuan et al. (2005) \\
\hline pT7- $\mathrm{H}_{6} \operatorname{TrxVirB11s}$ & $\begin{array}{l}\text { pT7- } \mathrm{H}_{6} \text { TrxFus with } 1083 \mathrm{bp} \text { Acc65I/HindIII fragment of VirB11 from } \\
\text { B. suis }\end{array}$ & This work \\
\hline pT7-7StrepIIVirB1sp & pT7-StrepII with 654 bp Acc65I/PstI fragment of virB1 from B. suis & This work \\
\hline pET21BC & $\begin{array}{l}\mathrm{Carb}^{\mathrm{R}} \text {, pET21b derivative with a DNA fragment encoding the S. pombe } \\
\text { ribosome-binding site and SRB11 cloned with SalI/NotI into pET21b } \\
\text { (with two RBS in the polylinker) for T7-driven bicistronic expression; } \\
\text { determines His } 6 \text {-tagged C-terminus of second ORF }\end{array}$ & This work \\
\hline pET24dVirB1spHis $_{6}$ & $\mathrm{Kan}^{\mathrm{R}}$, pET24d with 654 bp NcoI/NotI B. suis virB1 fragment & This work \\
\hline pET21BCVirB8sp-VirB1spHis 6 & $\begin{array}{l}\text { pET21BC with } 524 \text { bp NheI/EcoRI virB8 and } 654 \text { bp NcoI/NotI B. suis } \\
\text { virB1 fragment downstream }\end{array}$ & This work \\
\hline pET21BCVirB9sp-VirB1spHis 6 & $\begin{array}{l}\text { pET21BC with } 776 \text { bp virB9 and } 654 \text { bp B. suis virB1 fragment } \\
\text { downstream }\end{array}$ & This work \\
\hline pET21BCVirB10sp-VirB1spHis 6 & $\begin{array}{l}\text { pET21BC with } 1011 \text { bp virB10 and } 654 \text { bp B. suis virB1 fragment } \\
\text { downstream }\end{array}$ & This work \\
\hline pET21BCVirB8sp & $\begin{array}{l}\text { pET21BCVirB8sp-VirB1spHis }{ }_{6} \text {, virB1 gene removed with SalI/XhoI } \\
\text { and religated }\end{array}$ & This work \\
\hline pET21BCVirB9sp & $\begin{array}{l}\text { pET21BCVirB9sp-VirB1spHis } 6 \text {, virB1 gene removed with SalI/XhoI } \\
\text { and religated }\end{array}$ & This work \\
\hline pET21BCVirB10sp & $\begin{array}{l}\text { pET21BCVirB10sp-VirB1spHis } 6 \text {, virB1 gene removed with SalI/XhoI } \\
\text { and religated }\end{array}$ & This work \\
\hline
\end{tabular}


Table 2. PCR primers

\begin{tabular}{|c|c|c|}
\hline Primer & Sequence $^{*}$ & Vector cloned in \\
\hline VirB1s-3' & 5'-CGATCTGCAGTTAGAAAACAACTACGCCGTCCG-3' & \\
\hline VirB7s-3' & 5'-CCGGCTGCAGTTAGTCCTCGTAAGTGTCAACGG-3' & \\
\hline VirB11s-5 & 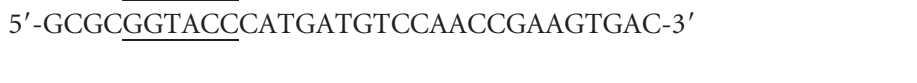 & $\begin{array}{l}\text { pT7-7StrepII and } \\
\text { pT7- } \text { H }_{6} \text { TrxFus }\end{array}$ \\
\hline Srb11-5 & $\begin{array}{l}\text { 5'-GGGGGGGTCGACAATAATTTTGTTTAACTTTAAGAAGGAGATATA- } \\
\text { CCATGGGTATGGCAGCAAATTACTGGGCCTCTAG-3' }\end{array}$ & pET21b \\
\hline Srb11-3' & 5' -GGGCCCGGGGCGGCCGCTTCAATATCCTCAAAATAATAAATAG-3' & \\
\hline VirB1sBC5': & 5'-GCGCGCCCATGGCAGCAATCGTGCAGGTCGAGT-3' & $\mathrm{pET} 21 \mathrm{BC} / \mathrm{pET} 24 \mathrm{~d}$ \\
\hline VirB1sBC3': & 5'-GACTGCGGCCGCGAAAACAACTACGCCGTCCG-3' & \\
\hline VirB10sBC5': & 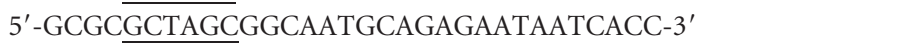 & pET21BC \\
\hline VirB10sBC3': & 5'-GCGCGAATTCTCATTGCAGGTTCTCCCCGGGC-3' & \\
\hline
\end{tabular}

${ }^{*}$ Restriction sites are underlined and Shine-Dalgarno motifs are in italics.

restriction sites. This placed virB1 in-frame with the vector sequence encoding the C-terminal $\mathrm{His}_{6}$-tag. A series of constructs for the expression of non-tagged proteins was generated to serve as controls, as these proteins did not bind avidly to affinity columns. Based on the pET21BC bicistron vectors, three constructs that served as negative controls were created by excision of virB1 with SalI/XhoI and religation of the vector. Untagged VirB8sp, VirB9sp or VirB10sp were produced upon induction of expression from these vectors.

Strep-Tactin Sepharose chromatography. Bacterial cells were resuspended in 4-8 ml StrepII buffer (S2B) without DTT (300 mM $\mathrm{NaCl}, 100 \mathrm{mM}$ Tris/HCl, $1 \mathrm{mM}$ EDTA, pH 7.0) and with $0.5 \mathrm{mM}$ PMSF and passed three times through a French pressure cell (Aminco) at 18000 p.s.i. The lysate was centrifuged (SS34 rotor, 25 min, 13000 r.p.m. at $4{ }^{\circ} \mathrm{C}$ ) to remove cell debris and unbroken cells, and the N-terminal StrepII-fusion protein was purified with a $1 \mathrm{ml}$ Strep-Tactin Superflow column following the instructions of the manufacturer (IBA), using $2 \cdot 5 \mathrm{mM}$ desthiobiotin in the elution buffer. The fractions were subsequently purified by size-exclusion chromatography using S2B at a flow rate of $0.5 \mathrm{ml} \mathrm{min}^{-1}$. Superdex 75 or Superdex 200 (Amersham Pharmacia Biosciences) was used depending on the molecular mass of the protein. The samples were dialysed for $>12 \mathrm{~h}$ against 1 litre of PSB (S2B with $50 \%$ glycerol) in dialysis tubing (Visking, Roth) at $4{ }^{\circ} \mathrm{C}$ and were stored at $-20^{\circ} \mathrm{C}$ until further use. Protein concentrations were determined using the Bradford dye binding assay (Bio-Rad) with bovine serum albumin as a reference.

Immobilized metal chelate affinity chromatography (IMAC). Cells were lysed in S2B without DTT $(0.5 \mathrm{mM}$ PMSF) and centrifuged as described above; the supernatant was applied to an HPLC system (Äkta Purifier, Amersham Pharmacia Biotech) with a $5 \mathrm{ml}$ $\mathrm{Co}^{2+}$-charged IMAC column (Talon Superflow, Clontech). His $6^{-}$ tagged recombinant protein was eluted according to a step-gradient protocol. At a flow rate of $0 \cdot 5-1 \cdot 0 \mathrm{ml} \mathrm{min}{ }^{-1}$ the column was first washed for 5 column volumes. Then, a stringent washing step with $20 \mathrm{mM}$ imidazole proceeded for $2 \cdot 5$ column volumes, before $150 \mathrm{mM}$ imidazole was applied to the column for $2 \cdot 5$ column volumes. Both the stringent wash fractions and the elution fractions were collected in $2 \mathrm{ml}$ aliquots, followed by gel filtration, dialysis in PSB and determination of the protein concentrations as described above.

Strep-Tactin Sepharose pull-down assay. Samples $(10 \mu \mathrm{l})$ of purified StrepII-tagged proteins ( $5 \mathrm{pmol}^{-1}$ in PSB) were incubated with $20 \mu \mathrm{l}$ Strep-Tactin Sepharose (50\% suspension in S2B, IBA) for $15 \mathrm{~min}$. Then $80 \mu \mathrm{l} \mathrm{S} 2 \mathrm{~B}$ and $10 \mu \mathrm{l} \mathrm{His}{ }_{6}$ TrxA-fusion protein $\left(5 \mathrm{pmol} \mu \mathrm{l}^{-1}\right.$ in PSB) were added. After 15 min incubation at room temperature, the Sepharose matrix was sedimented (centrifugation at 13000 r.p.m., $2 \mathrm{~min}$ ) and washed three times with $500 \mu \mathrm{l} \mathrm{S2B}$. Bound proteins were eluted with $35 \mu \mathrm{ll} \mathrm{mM}$ biotin followed by sedimentation of the matrix, mixing of the supernatant with 1 vol. Laemmli sample buffer, SDS-PAGE, Western blotting and analysis with VirB protein-specific antisera.

Gel filtration chromatography. Samples generated by affinity chromatography (1 ml maximum) were loaded onto Superdex 200 or Superdex 75 gel filtration columns in S2B; the flow rate was

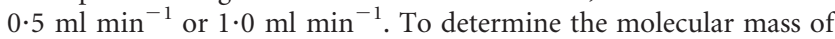
proteins, the columns were calibrated with the Gel Filtration Calibration Kit (Amersham Pharmacia Biotech), which uses reference proteins in the range between 13.7 and $669 \mathrm{kDa}$.

SDS-PAGE and Western blotting. Proteins were separated in denaturing SDS gels using the Laemmli system (Laemmli, 1970) followed by transfer to PVDF membranes (Immobilon-P, Millipore) in a vertical blot device (Trans Blot Cell, Bio-Rad; blot buffer $192 \mathrm{mM}$ glycine, $25 \mathrm{mM}$ Tris, $20 \%$ methanol) at $90 \mathrm{~V}$ for $1 \mathrm{~h}$ or $30 \mathrm{~V}$ for 16 h (Harlow \& Lane, 1988). Proteins attached to peptide array membranes (see below) were transferred in a semi-dry blot device (Fast-Blot, Biometra) onto PVDF membranes following a specialized protocol, followed by regeneration of the membrane as suggested by the manufacturer (Jerini). Proteins were detected with goat antirabbit IgG-HRP (Bio-Rad), a chemiluminescence detection system (Lumi Light, Roche Diagnostics) and X-ray film (Harlow \& Lane, 1988). 
Peptide array experiments. The entire sequence of VirB1sp from B. suis (GenBank accession no. NP_699276) without the signal peptide was displayed on a cellulose membrane as seventy 13-mers, covalently bound at the $\mathrm{C}$-terminus and with $\mathrm{N}$-terminal acetylation, shifting three amino acid positions each time, beginning with peptide 1 (AAIVQVESGFNPY), peptide 2 (VQVESGFNPYAIG), etc., to peptide 70 (PPGKDNTDGVVVF). The protocol for 'Mapping of discontinuous epitopes' from the manual of the supplier (Jerini) was followed. The peptide array membrane, which features all possible linear epitopes of VirB1sp, was preincubated for $30 \mathrm{~min}$ in TBS-T (20 mM Tris/HCl; $137 \mathrm{mM} \mathrm{NaCl} ; 0 \cdot 1 \%$ Tween-20; pH 8.0), transferred into blocking solution (Roche) for $1 \mathrm{~h}$, washed again with TBS-T for $10 \mathrm{~min}$ and then incubated in blocking solution containing 1-5 $\mathrm{g} \mathrm{ml}^{-1}$ of different proteins (StrepIIVirB1sp, StrepIIVirB8sp, StrepIIVirB9sp or StrepIIVirB11s) for $12 \mathrm{~h}$ at $4{ }^{\circ} \mathrm{C}$. Before transfer of the attached proteins onto PVDF membranes with a semi-dry blot device (see above), the peptide array membrane was washed three times in TBS-T for $10 \mathrm{~s}$ to remove non-specifically bound protein.

Generation of polyclonal antisera. Soluble StrepIIVirB11s and $\mathrm{H}_{6}$ TrxAVirB7 were purified by affinity chromatography as described above, whereas StrepIIVirB1sp was obtained from a preparation of inclusion bodies, separated by SDS-PAGE, excised from the gel and subjected to electroelution. Approximately $0.5 \mathrm{mg}$ of each protein was lyophilized and used for immunization of rabbits (BioGenes) to generate specific antisera. The other antisera used in this study were described elsewhere (Yuan et al., 2005).

Graphical data processing. To capture images of polyacrylamide gels and chemoluminogramms, they were digitized using a UMAX UTC-6400 scanner, followed by processing with Photoshop 6.0 (Adobe) and Canvas 7.0 (Deneba Systems).

Protein sequence analysis. The CLUSTAL W (version 1.82) algorithm for multiple sequence alignment (Higgins, 1994) (http:// www.ebi.ac.uk/clustalw) or EMBOss for alignment of two less conserved amino acid sequences (Needleman \& Wunsch, 1970; Smith \& Waterman, 1981) (http://www.ebi.ac.uk/emboss/align) were applied. Sequence information was processed with NORSp (Liu \& Rost, 2003) (http://cubic.bioc.columbia.edu/services/NORSp) in order to discover long regions without regular secondary structure. Predictions of secondary structure were obtained with the PHD algorithm (Rost, 1996) (http://www.embl-heidelberg.de/predictprotein). To create a conservation plot of sequence alignment, the alignment data were transferred to the AMAS server (Livingstone \& Barton, 1993) (http://barton.ebi.ac.uk/servers/amas_server.html) using standard default values. All structure images were generated with DINO 9.0 (http://cobra.mih.unibas.ch/dino/intro.php).

\section{RESULTS}

\section{Purification and characterization of affinity- tagged B. suis VirBs proteins}

To analyse interactions of VirB1s with B. suis VirBs proteins the region of virB1 encoding the predicted periplasmic domain of the protein without signal peptide was PCRamplified and cloned into pT7-7-StrepII for expression with an N-terminal StrepII peptide (StrepIIVirB1sp) or into pET24d for expression with a C-terminal $\mathrm{His}_{6}$ tag (VirB1spHis ${ }_{6}$ ). The gene encoding full-length VirB11s was PCR-amplified and cloned into pT7-7StrepII for expression with an N-terminal StrepII peptide (StrepIIVirB11s). Similar clones for expression of StrepIIVirB8sp, StrepIIVirB9sp and StrepIIVirB10sp were described previously
(Yuan et al., 2005). The proteins were overproduced and purified via affinity columns, followed by gel filtration over a Superdex 200 column for further purification and analysis of their molecular masses. Analysis with a specific antiserum showed that both $\mathrm{N}$ - and C-terminally tagged variants of VirB1sp eluted in a broad molecular mass range; we have indicated the predicted sizes of monomers, dimers, tetramers and hexamers in Fig. 1. The relative distribution between the different forms varied between experiments and the hexameric form was not always prominent, suggesting a dynamic equilibrium between different multimeric forms. In addition, large portions of VirB1sp (70-80\%) eluted in the void volume in high-molecular-mass complexes (Fig. 1). The elution of lytic transglycosylases in high-molecularmass complexes has also been observed by others and was shown to be due to binding of the proteins by GroEL (Zahrl et al., 2005). The possibility that GroEL binds to VirB1sp was therefore assessed by Western blot analysis of the samples eluted from the gel filtration column using specific antisera, and we indeed detected co-elution of VirB1sp variants with GroEL in the void volume (not shown). Analysis of the molecular masses of the other VirBs proteins by gel filtration showed that they eluted as apparent monomers (StrepIIVirB8sp and StrepIIVirB9sp), dimers (StrepIIVirB10sp) or hexamers (StrepIIVirB11s) (Table 3). Varying degrees of high-molecular-mass aggregates eluting in the void volume of gel filtration columns were observed in all cases. The amounts varied in different overexpression experiments and could not be reduced by the addition of DTT or increased $\mathrm{NaCl}$ concentrations (not shown). Whereas we cannot exclude that these aggregates are of physiological relevance,
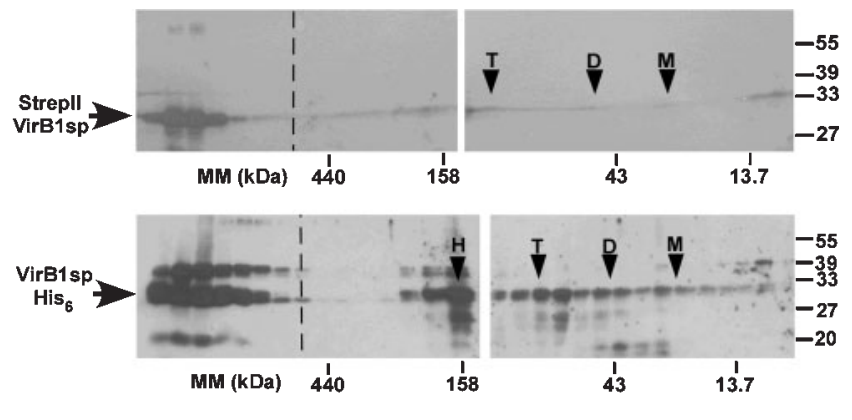

Fig. 1. Analysis of multimer formation of StrepllVirB1sp and $\mathrm{VirB1}_{\mathrm{spHis}}$ by gel filtration: Superdex 200 gel filtration analysis of the molecular masses of tagged VirB1sp variants after overproduction and purification by affinity chromatography. After gel filtration, the proteins in the eluted $2 \mathrm{ml}$ fractions were separated by SDS-PAGE followed by Western blotting and detection with VirB1sp-specific antiserum. A dashed line marks the Superdex 200 void volume at $>600 \mathrm{kDa}$. Arrowheads point to the expected sizes of monomeric (M), dimeric (D) tetrameric $(\mathrm{T})$ and hexameric $(\mathrm{H})$ proteins. Molecular masses of SDS-PAGE marker proteins are given on the right in $\mathrm{kDa}$; the elution of gel filtration reference proteins with their associated molecular masses (MM) from the Superdex 200 column is indicated below the chemoluminograms. 
Table 3. Molecular masses of StrepllVirB proteins

\begin{tabular}{|lccc|}
\hline Protein analysed & $\begin{array}{c}\text { Expected mass of } \\
\text { the monomer }(\mathbf{k D a})\end{array}$ & $\begin{array}{c}\text { Mass determined } \\
\text { by gel filtration }(\mathbf{k D a})\end{array}$ & $\begin{array}{c}\text { Tertiary structure } \\
\text { determined by } \\
\text { gel filtration }\end{array}$ \\
\hline $\begin{array}{l}\text { StrepIIVirB1sp } \\
\text { and VirB1spHis }\end{array}$ & 27 & Various to 160 & $\begin{array}{c}\text { Monomer, dimer, } \\
\text { tetramer, hexamer }\end{array}$ \\
StrepIIVirB8sp & 22 & 26 & Monomer \\
StrepIIVirB9sp & 33 & 41 & Monomer \\
StrepIIVirB10sp & 43 & 92 & Dimer \\
StrepIIVirB11s & 41 & 242 & Hexamer \\
\hline
\end{tabular}

we did not consider this as very likely and used only the lower-molecular-mass fractions for the following studies.

\section{Analysis of interactions by gel filtration}

As a first approach to assess interactions between purified StrepIIVirBs proteins, mixtures of these proteins were coincubated to allow complex formation. The samples were then subjected to gel filtration chromatography over a Superdex-200 column. Complex formation was assumed to lead to shifts of the elution volumes, but we did not observe changes in any case. This suggested that these proteins do not interact, or that the affinities are not high enough to form complexes stable during gel filtration (not shown).

We subsequently followed an alternative strategy based on the observation that recombinant proteins produced in E. coli are often insoluble or misfolded, and one reason for this is that their natural binding partners are absent. One method to circumvent this problem is the co-expression of the genes encoding such proteins together with genes encoding potential interaction partners (Lutzmann et al., 2002). To this end, the potential interactions of VirB1sp with other components of the B. suis T4SS were tested by expression from vectors encoding C-terminally tagged VirB1spHis $_{6}$ fusion proteins and their putative interaction partners VirB8sp, VirB9sp or VirB10sp. In order to coproduce $\mathrm{His}_{6}$-tagged VirB1sp with putative interaction partners, vector pET21BC was constructed to permit expression of bicistronic mRNAs. The first ORF of the pET21BC series encoded the putative interaction partners VirB8sp, VirB9sp and VirB10sp, and the second one VirB1spHis $_{6}$. In addition, a series of pET21BC-derived monocistronic constructs for the expression of the nontagged interaction partners VirB8sp, VirB9sp or VirB10sp without VirB1spHis 6 was generated to serve as controls.

The proteins encoded on the pET21BC-derived vectors were overproduced in E. coli, followed by cell lysis, purification over a $\mathrm{His}_{6}$-tag-specific affinity column and Superdex 200 gel filtration for analysis of complex formation. In spite of the two-step separation procedure, the untagged VirBsp proteins from both monocistronic and bicistronic expression experiments were detected in the gel filtration eluates, indicating that VirB8sp, VirB9sp and VirB10sp had non-specific binding affinity to the column. In order to distinguish between co-elution due to similar molecular masses and co-elution as an effect of an interaction, we compared the elution after expression from a bicistronic expression vector with that after expression from a monocistronic vector. VirB8sp eluted as a monomer in fractions 24-28 from the gel filtration in both cases, and VirB1spHis eluted in fractions 17-21, supposedly the tetrameric and dimeric form (Fig. 2a). The co-expression with virB8 thus had no apparent effect on the elution of VirB1spHis and vice versa, suggesting that these two proteins did not interact. When VirB1spHis ${ }_{6}$ was produced from the bicistronic vector with VirB10sp it eluted in fractions 17-19, which corresponded to the molecular mass expected for the tetramer (Fig. 2c). VirB10sp produced in strains carrying the monocistronic as well as the bicistronic vector eluted in fractions 17-19 as a dimer. As the molecular masses of VirB1spHis $_{6}$ and VirB10sp were very similar, this experiment did not give any evidence for an interaction. In contrast, when VirB1spHis ${ }_{6}$ was produced from the bicistronic vector with VirB9sp it eluted from the column in three forms (Fig. 2b). First, it was detected in fractions 6-9, representing a large complex that had a lower molecular mass than that of VirB1spHis ${ }_{6}$ when it was expressed from a monocistronic vector. Second, it eluted in fractions 15-17, corresponding to a complex markedly larger than the hexamer of $160 \mathrm{kDa}$, and third, it was detected in fractions 21-23, corresponding to a size between the dimer and tetramer. VirB9sp eluted in fractions 6-9 and 15-17 but the largest portion of the protein was monomeric (fractions 22-25). In contrast, when VirB9sp was expressed from the monocistronic vector, it predominantly eluted in fractions $22-25$, which corresponded to the supposed monomer. It is evident from the comparison of the elution profiles that the co-expression with VirB9sp affected the oligomeric state of VirB1spHis $_{6}$ and vice versa. Whereas this method did not permit the unambiguous identification of hetero-oligomer formation, the results support a direct interaction between the two proteins. Alternative methods were employed in the following to further assess this possibility.

\section{Analysis of interactions by affinity precipitation}

An alternative way to demonstrate protein-protein interactions is a pull-down assay that exploits the affinity of the 
(a)

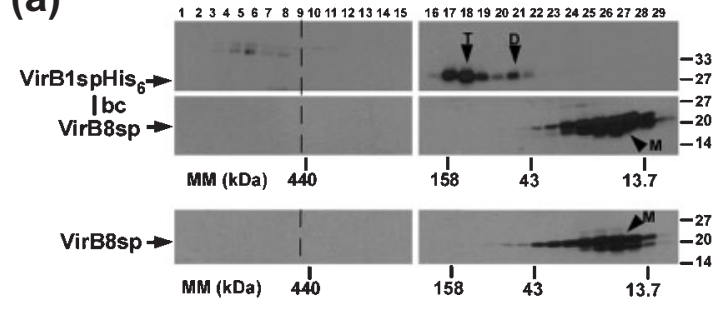

(b)

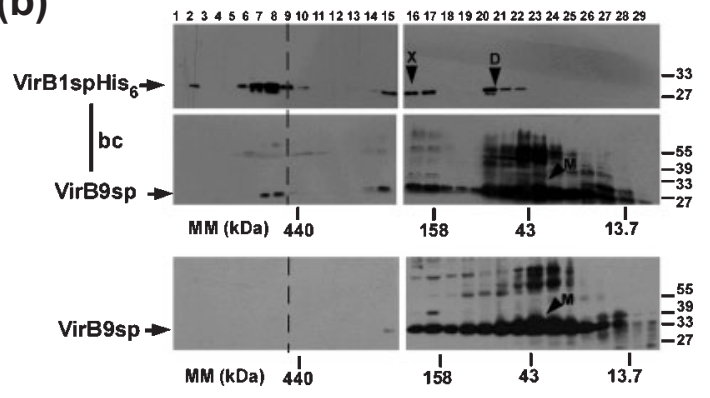

(c)

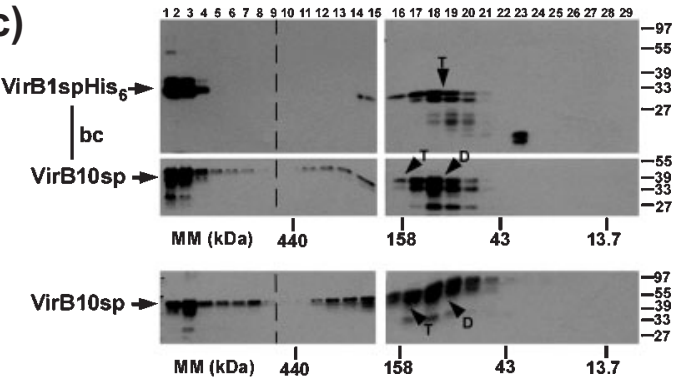

Fig. 2. Coelution of VirB1spHis 6 with other T4SS components: Superdex 200 gel filtration analysis of the molecular masses of VirB8sp (a), VirB9sp (b) and VirB10sp (c) expressed alone or from bicistronic vectors (bc) together with VirB1spHis ${ }_{6}$ after overproduction and purification by affinity chromatography. After gel filtration, the proteins in the eluted $2 \mathrm{ml}$ fractions were separated by SDS-PAGE followed by Western blotting and detection with specific antisera. Upper panels were probed with VirB1sp-specific antiserum, whereas lower panels were probed with antisera specific for VirB8sp (a), VirB9sp (b) and VirB10sp (c), respectively, as indicated by arrows. A dashed line marks the Superdex 200 void volume at $>600 \mathrm{kDa}$. Arrowheads point to the expected size of monomeric (M), dimeric (D) and tetrameric (T) proteins. VirB1spHis 6 was detected in a complex of typical molecular mass (X), which was larger than the hexamer, exclusively after co-expression with VirB9sp. Molecular masses of SDS-PAGE marker proteins are given on the right in $\mathrm{kDa}$; the elution of gel filtration reference proteins with their associated molecular masses (MM) from the Superdex 200 column is indicated below the chemoluminograms. All experiments were performed up to three times with similar results and the results of one set of representative experiments is shown.

StrepII tag for the Strep-Tactin Sepharose affinity matrix. The VirBs proteins that were tested for their capacity to bind StrepIIVirB1sp were affinity-purified as $\mathrm{His}_{6}$-tagged thioredoxin fusions ( $\mathrm{His}_{6} \mathrm{TrxA}$ ) and their co-precipitation with
StrepII-tagged VirB1sp bound to the affinity matrix was determined. His ${ }_{6}$ TrxAVirB7sp, which was not expected to interact with VirB1sp, was included as a negative control for non-specific binding of the His ${ }_{6}$ TrxA affinity tag. Equimolar mixtures of StrepIIVirB1sp were incubated with $\mathrm{His}_{6}$ TrxAVirB7sp, His ${ }_{6}$TrxAVirB8sp , His ${ }_{6}$ TrxAVirB9sp, His ${ }_{6}$ TrxAVirB10sp or $\mathrm{His}_{6}$ TrxAVirB11s, followed by sedimentation of the affinity matrix, washing and elution of StrepIIVirB1sp and attached binding partners with biotin, SDS-PAGE and Western blot analysis. His ${ }_{6}$ TrxAVirB7sp and His 6 TrxAVirB10sp did not co-precipitate with StrepIIVirB1sp, showing that these proteins did not interact under these conditions (Fig. 3). These results also demonstrated that the His $_{6}$ TrxA tag had no affinity for the matrix utilized here. A different observation was made in the case of $\mathrm{His}_{6}$ TrxAVirB8sp, which bound to the matrix irrespective of the presence of StrepIIVirB1sp. Since even extensive washing could not remove the protein from the Strep-Tactin matrix, it was impossible to use this method to assess this interaction. Co-fractionation with StrepIIVirB1sp was demonstrated in the case of $\mathrm{His}_{6}$ TrxAVirB9sp and His ${ }_{6}$ TrxAVirB11s (Fig. 3). These results substantiate the interaction of VirB1sp with VirB9sp observed above and suggest that VirB11s may be another interaction partner. Since our experiments gave evidence for interactions of VirB1sp with VirB9sp and VirB11s, and VirB8sp may also interact with VirB1sp (Ward et al., 2002), we next analysed the binding site(s) on VirB1sp using peptide array experiments.

\section{Peptide array analysis of VirB1sp interactions}

The analysis of binding to peptide arrays constitutes a highresolution method to narrow down interaction site(s) on a protein, which has been used successfully for antibody epitope mapping and for the analysis of protein-protein interaction sites (Burns-Hamuro et al., 2003; Knoblauch et al., 1999; Llanos et al., 1999; Reimer et al., 2002; Reineke et al., 1999). The analysis of multiple binding partners of different binding affinities in parallel permits a direct comparison of the bound region(s) and enables positive and negative control experiments. To this end, the binding of StrepIIVirB8sp, StrepIIVirB9sp and StrepIIVirB11s to 70 peptides displayed on membranes (13 amino acids long, three amino acids overlap), which represented the entire sequence of the processed form of VirB1sp, was analysed. Three identical membranes were used in parallel experiments, and they retained StrepIIVirBs proteins in every case. Western blots from five independent experiments, which determined the binding of an interaction partner to specific peptides, were graphically superimposed to obtain a representative mean result (Fig. $4 \mathrm{a}-\mathrm{c}$ ). The intensity of the signal for each spot was categorized from 1 (weak) to 4 (very strong). By aligning the sequences of the bound peptides, which overlapped by three amino acids, it was possible to identify domains of VirB1 that bind the respective VirBsp protein (Fig. 4d). When more than one spot defined an interacting domain in the sequence, the highest value of signal intensity present was assigned to the entire domain. 


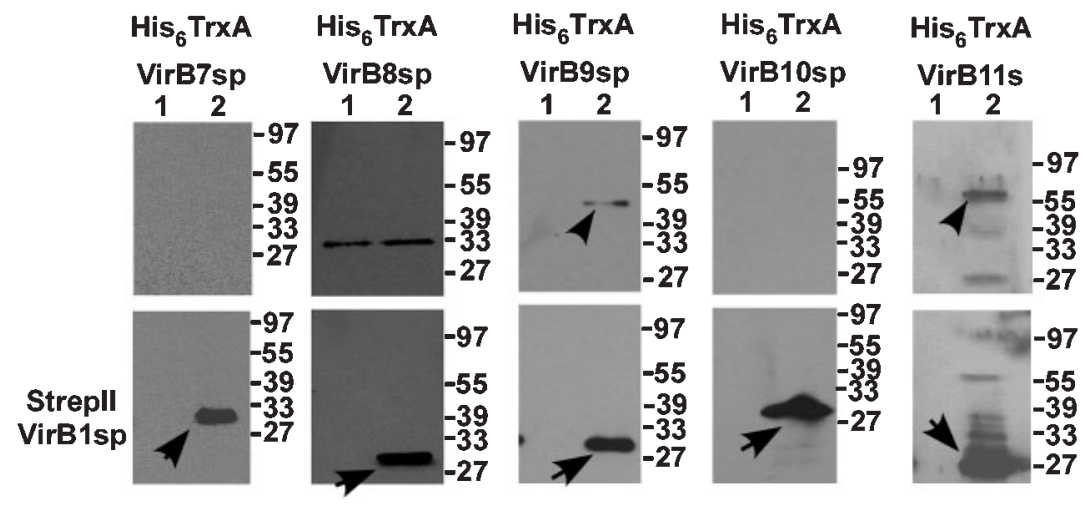

Fig. 3. Strep-Tactin Sepharose pull-down assay with StreplIVirB1sp. StreplIVirB1sp was preincubated with the Strep-Tactin matrix before addition of the indicated $\mathrm{His}_{6}$ TrxAVirBsp proteins. After washing three times, proteins were eluted from the matrix with $1 \mathrm{mM}$ biotin, and analysed by SDSPAGE, Western blotting and detection with specific antisera. Lower panels were probed with VirB1sp-specific antiserum, whereas upper panels were probed with antisera specific for VirB7sp, VirB8sp, VirB9sp, VirB10sp and VirB11s, respectively. Lanes 1 show the negative controls of only $\mathrm{His}_{6}$ TrxAVirBsp proteins incubated with the matrix; lanes 2 show the pull-down assays after co-incubation with StreplIVirB1sp. Arrows indicate StreplIVirB1sp sedimented with the matrix; arrowheads indicate cosedimenting interaction partners. Experiments were repeated five times and representative results are shown. Molecular masses of reference proteins are shown on the right in $\mathrm{kDa}$.

The three studied proteins StrepIIVirB8sp, StrepIIVirB9sp and StrepIIVirB11s bound to peptides from different regions of VirB1sp and the binding strengths were different, as indicated by the numbers of bound peptides and the signal intensity on the chemoluminogram. StrepIIVirB9sp bound to the highest number of VirB1sp peptides, but StrepIIVirB8sp and StrepIIVirB11s also bound to a defined set of VirB1sp peptides. To place this information in the context of the protein structure, a prediction of the VirB1sp secondary structure was done with the PHD algorithm and the proposed secondary structure is also shown in Fig. 4(ac). Weak binding of StrepIIVirB8sp occurred to a region Cterminal to the catalytic $\mathrm{Glu}^{27}$ residue, which is predicted to be a loop/ $\beta$-sheet region (Fig. 4a). StrepIIVirB8sp strongly interacted with another loop region $\mathrm{N}$-terminal to the $\mathrm{C}$ terminus. StrepIIVirB9sp bound to different amino acid stretches throughout the entire sequence of VirB1 (Fig. 4b). Interactions of intermediate strength occurred with three parts of the VirB1sp sequence that are only three amino acids long. StrepIIVirB9sp bound to the first three amino acids following the predicted signal peptide of VirB1, a loop region $\mathrm{C}$-terminal to a short $\beta$-sheet and $\mathrm{N}$-terminal to the conserved GIAQ motif, which is characteristic of all soluble lytic transglycosylases. An extended domain bound with intermediate strength by StrepIIVirB9sp was localized to the C-terminus of VirB1. Strong interactions with the amino acids $C^{127}-Y^{128}-Y^{129}$ were observed, which are predicted to participate in the formation of an $\alpha$-helix $\mathrm{N}$-terminal to the C-terminus. Very strong binding to a loop region shortly after the GIAQ motif and N-terminal to the start of the C-terminus was detected. In addition, two long amino acid sequence stretches in the C-terminus were strongly recognized by StrepIIVirB9sp (Fig. 4b). StrepIIVirB11s bound only to a limited set of peptides on the VirB1sp array membrane (Fig. 4c). A weak interaction of StrepIIVirB11s with the VirB1sp sequence was apparent in a loop/ $\beta$ sheet region C-terminal to the catalytic Glu ${ }^{27}$. Strong binding to an $\alpha$-helix/loop region and to a loop region in the amino acid sequence constituting the second half of the lytic transglycosylase domain was detected. A very strong interaction of StrepIIVirB11s was observed with a short loop region C-terminal to the GIAQ motif. These results further substantiated the interactions of VirB1sp with other T4SS components shown by different methods above. Interacting amino acids are often found in external loop regions, but a structure of VirB1sp was not available. To further assess the biological relevance of these interactions it was necessary to place the information on bound peptides in the context of a three-dimensional structure, and a model of the VirB1sp structure was generated next.

\section{Modelling of the VirB1 structure and localization of its VirB protein interaction site(s)}

To assess whether the sequence stretches of VirB1sp that interact with other proteins are spatially clustered and are conserved among different VirB1-like proteins, the X-ray structure of the soluble lytic transglycosylase Slt70 from $E$. coli was used as a model to approximate the structure of $B$. suis VirB1. To identify residues conserved among VirB1-like proteins, several of them were aligned with E. coli Slt70 (Fig. 5a). Next, the AMAS algorithm was used to assign values expressing the degree of conservation to all amino acid positions in a multiple sequence alignment, ranging from A (identical) to 8 (weakly conserved). One of the sequences in this alignment was a protein with known tertiary structure (here E. coli Slt70) and the other was B. suis 

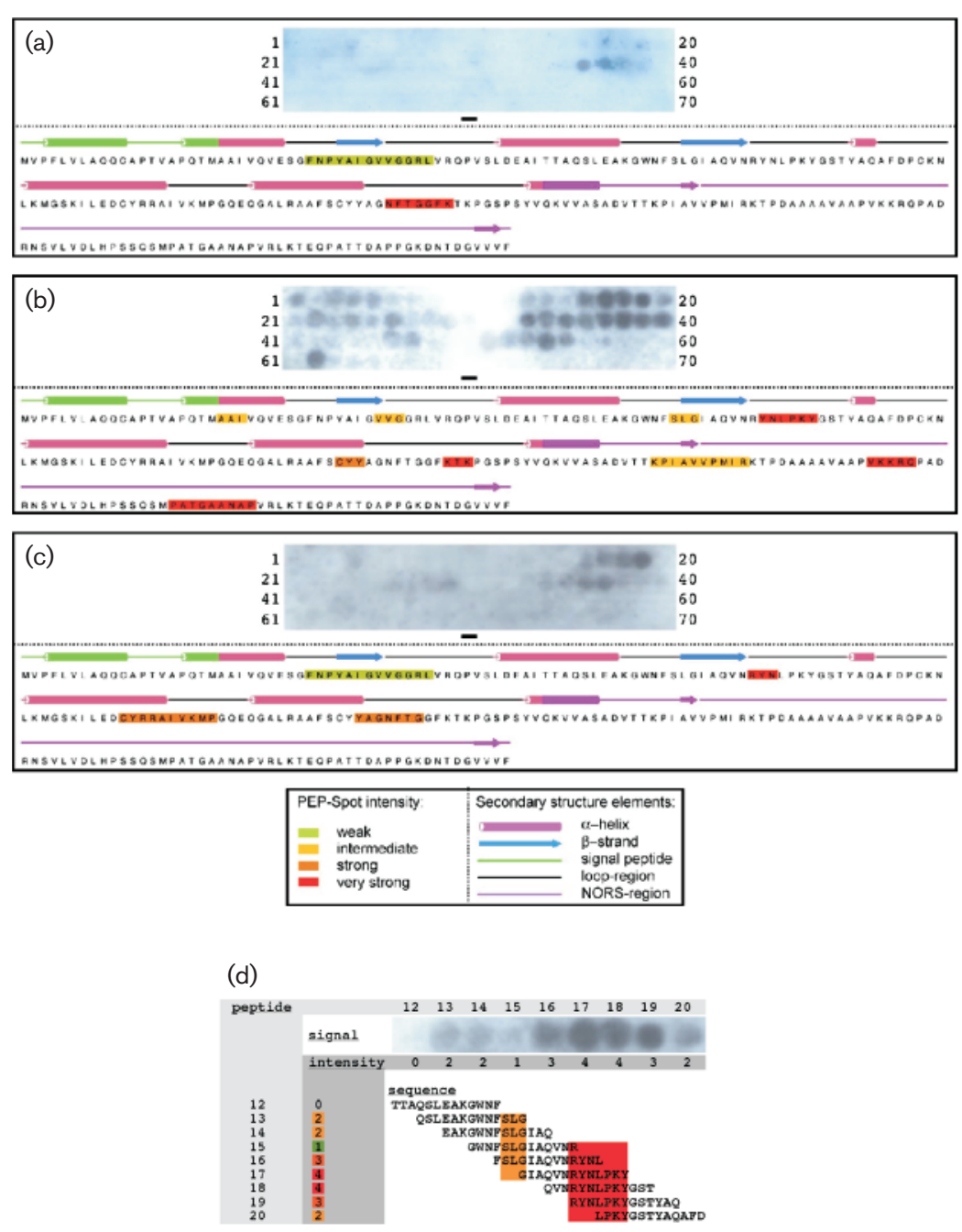

Fig. 4. Binding of StrepllVirB8sp, StrepllVirB9sp and StrepllVirB11s to VirB1sp peptide array membranes. Binding of tagged VirB8sp (a), VirB9sp (b) and VirB11s (c) to VirB1sp peptides spotted on membranes (pepspot arrays) was determined by Western blot transfer of the bound proteins to PVDF membranes, followed by detection with specific antisera. The chemoluminograms represent the results of five independent experiments, which were graphically superimposed. A colour code is used to indicate the intensity of VirB protein binding (pepspot intensity) for every stretch of the corresponding VirB1 sequence. Secondary structure elements and the proposed signal peptide of VirB1 as predicted by the PHD algorithm (Rost, 1996) are also displayed below the chemoluminograms. (d) Method of peptide array evaluation. Signals from the Western blot (as an example for illustration, an enlarged portion from the VirB1sp membrane incubated with StrepllVirB9sp is shown) were categorized by visual inspection, and the intensity values (1, weak, 2, intermediate, 3, strong, 4, very strong) assigned to a certain peptide number and colour. By alignment of the overlapping peptide, the sequence responsible for the observed signal (binding of StreplIVirB9sp) can be identified. In the case of 'RYNLPKY', shown here, the most intense signal was categorized as 4 (very strong), and this value and the corresponding colour (red) were assigned to the entire sequence stretch.
VirB1. The software DINO was next applied to create a map of conserved residues on the surface of the known threedimensional model (Fig. 5b, c). It is assumed that conserved patches on the surface are likely to interact either with partner proteins or substrate(s). Following the modelling we found that many of the amino acids conserved between VirB1 and Slt70 were not exposed on the surface but rather seem to stabilize the structurally conserved lysozyme fold. The most prominent conserved surface patch was the activesite cleft with the catalytic Glu residue, which is ubiquitously found throughout all lysozyme-like enzymes (Fig. 5b, c). The C-terminus of VirB1s did not align with Slt70 and it is thus not part of the model.

Next, the model was used to localize the interaction sites with StrepIIVirB8sp, StrepIIVirB9sp and StrepIIVirB11s on the surface of the VirB1sp model (Fig. 5d). The peptide array experiments yielded signals of different intensities corresponding to peptides featuring parts of the VirB1sp sequence. The corresponding sequences were aligned, and defined regions of interaction. For each interacting region, the intensity of the signals constituting it was categorized into four classes, from 'weak' to 'very strong'.
This classification did not compare signal intensities between peptide array experiments with different proteins and therefore, a 'strong' interaction of VirB1sp peptides with StrepIIVirB8sp might be classified as 'intermediate' in a peptide array with StrepIIVirB9sp. The studies are therefore semi-quantitative and need to be followed up by more quantitative methods in future. StrepIIVirB8sp and StrepIIVirB9sp apparently interacted with surface-exposed loop regions of VirB1sp that are most probably situated on different sides of the protein, and StrepIIVirB11s may bind to a part of the protein that connects these two (Fig. 5d). Interestingly, both StrepIIVirB9sp and StrepIIVirB11s bound strongly to peptides representing a not well-conserved part of the protein C-terminal to the second region, which is probably required for enzymic activity (boxed in Fig. 5a). Similarly, both StrepIIVirB8sp and StrepIIVirB9s bound strongly C-terminal to the third region implicated in enzyme activity of lytic transglycosylases (boxed in Fig. 5a). Most of the proposed interactions between VirB1sp and StrepIIVirB9sp could not be visualized in this model since they map to the C-terminal region of VirB1sp, which lacks a counterpart in Slt70. Nevertheless, the identification of binding sites for other T4SS components in a structure 
(a)

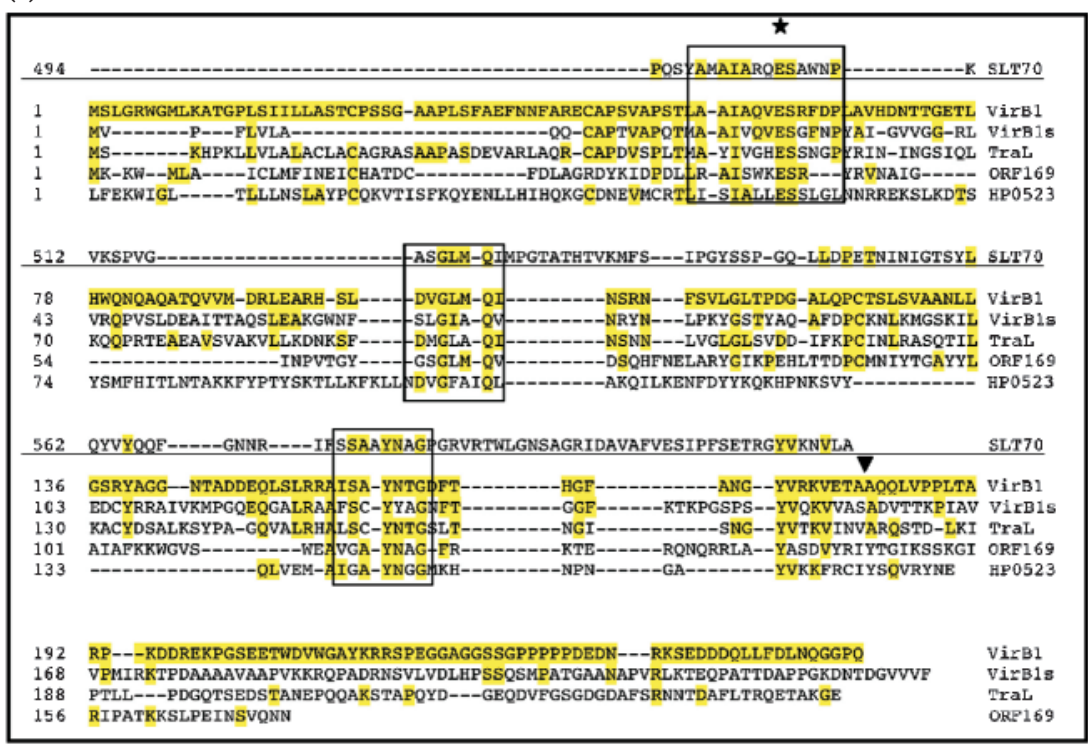

(b)

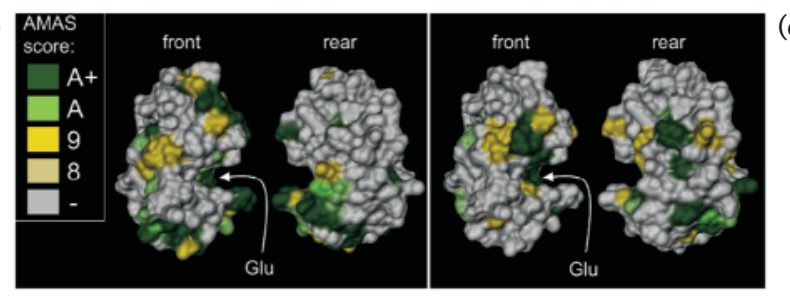

(d)

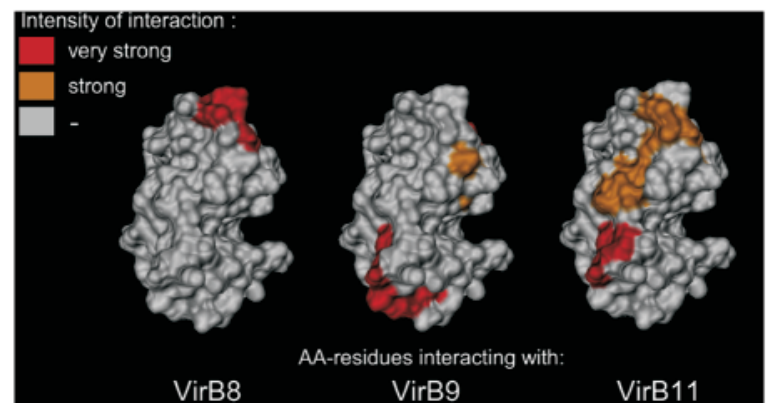

Fig. 5. Prediction of conserved surface residues in different lytic transglycosylases and regions of VirB1sp peptide arrays bound by StreplIVirBs proteins. (a) Multiple sequence alignment of VirB1-like proteins. Residues identical to $A$. tumefaciens VirB1 are shaded; conserved residues implicated in the enzymic activity are indicated by black frames. The putative active-site Glu is labelled with a star; the cleavage site of $A$. tumefaciens VirB1 is indicated with a black arrowhead. The alignment of the VirB1 orthologues was generated with the MegAlign program, the SLT domain of $E$. coli Slt70 was aligned to B. suis VirB1 with EMBOss (Needle algorithm) and fitted into the alignment. Amino acids conserved between the lytic transglycosylase domain of B. suis VirB1 and E. coli Slt70 (b) or A. anser LysG and E. coli Slt70 (c) are displayed on the surface of the SIt70 soluble lytic transglycosylase domain. The results from an EMBOSS alignment were processed and submitted to the AMAS server to validate the degrees of conservation. Identical residues in each of the two pairs are shown in dark green $[\mathrm{A}+]$, high similarity $[A]$ in green, and lower similarity is in yellow [9] and light yellow [8]. The figure was prepared with DINO and the active-site Glu residue is indicated. (d) View of the Slt70 lytic transglycosylase domain surface structure taken as a model for VirB1sp. Interactions with StreplIVirB8sp, StrepllVirB9sp and StreplIVirB11s as defined by the peptide array experiments are shown. A grey colour indicates no significant interaction; orange colour indicates strong and red colour very strong interaction. model of VirB1sp constitutes a substantial advance in our understanding of the biological role of this protein. Our work lays the foundation for detailed structure-function analyses in future.

\section{DISCUSSION}

A role of VirB1 as nucleation centre for T4SS assembly has been suggested before, but hitherto, the interactions of VirB1 with T4SS components had only been demonstrated in the yeast dihybrid system (Ward et al., 2002). Here we have directly studied the interaction of VirB1sp with most of its proposed interaction partners. This analysis was possible because the B. suis T4SS components are more amenable to biochemical experiments than their counterparts from the A. tumefaciens T4SS. Many of its components are relatively easy to purify, because in contrast to those from $A$. tumefaciens, they can be overexpressed in a soluble form with high yield, followed by affinity purification. This feature was exploited to conduct biochemical experiments to assess the suggested interactions. A portion of StrepIIVirB1sp and VirB1spHis ${ }_{6}$ eluted from gel filtration columns in a multimeric protein complex (or aggregate) together with GroEL. We did not pursue the analysis of this complex further, as we did not consider it as the physiologically relevant form. Apart from that, VirB1spHis 6 was mainly present in the dimeric and tetrameric state and with reduced prominence as a hexamer. A larger portion of StrepIIVirB1sp was present in the high-molecular-mass complex, but it was also detected as lower-molecular-mass multimer. The available data suggest that StrepIIVirB1sp forms homomultimeric assemblies, probably dimers and tetramers. The molecular sieving effect of the murein layer prohibits diffusion of globular proteins or protein assemblies larger than $55 \mathrm{kDa}$ (Höltje, 1998). Since the permeation of the 
murein layer might be important for the function of lytic transglycosidase in the periplasm, the formation of dimers (approx. $50 \mathrm{kDa}$ ) appears to be more plausible in the natural biological context than the formation of tetramers (approx. $100 \mathrm{kDa}$ ), which may not permeate the murein layer.

The purified B. suis VirB proteins were then used to systematically assess the predicted interactions with VirB1sp. Some of those interactions were confirmed here, whereas others were only weak or not observed. Using co-elution and pull-down assays we did not get any evidence for an interaction between VirB1sp and VirB8sp or VirB10sp, which had been predicted from a previous yeast two-hybrid study (Ward et al., 2002). In contrast, data from the peptide array experiment suggested that a set of VirB1sp peptides interacted with StrepIIVirB8sp, which supports the notion that these two proteins interact at least transiently. The evidence is not as substantial as that in case of StrepIIVirB9sp discussed below, suggesting that the interaction is weaker and/ or may need additional interaction partners. These results are in line with the supposed role of VirB8 as nucleation factor, which undergoes transient interactions with many T4SS components (Kumar \& Das, 2001; Yuan et al., 2005). The in vitro data therefore argue against the postulated mechanism implying the VirB8-VirB1 interaction as key step for T4SS assembly (Ward et al., 2002), but we can not rule out that the interaction is stronger in vivo.

Similar to VirB8sp, we obtained evidence for an interaction of the hexameric ATPase VirB11s with VirB1sp using peptide array as well as pull-down experiments. The interaction between VirB1sp and VirB9sp was demonstrated using a variety of different assays such as a pull-down assay and coelution of the two proteins following their co-expression in a bicistronic construct. Peptide array experiments identified several StrepIIVirB9sp-interacting peptides throughout the VirB1sp sequence, and a considerable portion of them was in the C-terminus. Whereas this might argue for nonspecific binding of StrepIIVirB9sp to the VirB1sp-derived peptides, we do not favour this interpretation for the following reasons. First, we have tested the binding of StrepIIVirB9sp to a pepspot membrane displaying the VirB5sp sequence, and, in line with other results showing that it does not bind strongly to VirB5sp, no non-specific binding to the membrane was observed (unpublished observations). Second, we observed non-specific binding of StrepIIVirB1sp as reflected by binding to most peptides on the VirB1sp pepspot membrane. Both the extent and strength of the signal were drastically elevated as compared to the relatively modest signal of bound StrepIIVirB9sp (unpublished observations). Finally, the peptide array data are very much consistent with the results of a previous study in intact cells, which demonstrated the interaction of the VirB1 C-terminus with VirB9 in A. tumefaciens (Baron et al., 1997). We therefore conclude that the binding by StrepIIVirB9sp to many peptides of VirB1sp is likely to be of biological relevance.

The interaction with VirB9sp via the C-terminus is intriguing and this domain appears to play an important role for the functionality of the protein. Sequence analyses of a number of VirB1 homologues demonstrated special properties of the $\mathrm{C}$-terminal part. The processed $A$. tumefaciens VirB1 C-terminus VirB1* (Baron et al., 1997) and also the C-termini of B. suis VirB1 and pKM101 TraL were classified as NORS regions. These are regions of more than 70 amino acids in length that show less than $12 \%$ secondary structure elements and an amino acid composition different from loop regions. It was demonstrated that these very flexible regions show similar degrees of conservation as other domains in similar proteins, and that they are more abundant in proteins with functions as regulators or transcription factors than in those with functions in biosynthesis or energy metabolism-related proteins (Liu \& Rost, 2003). This implies important functional roles, most likely for transient protein-protein interactions with different partners. Only $4 \%$ of all prokaryotic proteins contain NORS regions, and among the VirB proteins from $A$. tumefaciens and B. suis, channel component VirB10, which is supposedly involved in a high number of interactions (Cascales \& Christie, 2003, 2004), is the only other protein that possesses such a region. Most interacting amino acid stretches identified here constitute loop or NORS regions, which are especially suited for establishing transient protein-protein interactions, suggesting that the interaction sites are biologically relevant.

To assess the biological relevance of the interactions and binding site(s) identified here, we pursued a modelling approach based on the known X-ray structure of a soluble lytic transglycosylase enzyme. To date, the structures of 18 murein-lytic lysozymes from different organisms have been solved. In addition, the three structures of the lytic transglycosylases LaL from bacteriophage $\lambda$, Slt35 and Slt70 (both from E. coli) are also available (Leung et al., 2001; Thunnissen et al., 1994; van Asselt et al., 2000). The enzymic action of lysozymes and soluble lytic transglycosylases differs, but the protein fold is highly conserved (Mushegian et al., 1996). Sequence comparison of B. suis VirB1 with two murolytic enzymes, whose X-ray structure was known, yielded intriguing results. The soluble lytic transglycosylase portion of Slt70 from E. coli ranges from amino acid P494 to A620 and has a significant degree of sequence similarity to $B$. suis VirB1 (identical, $23 \cdot 1 \%$; similar, $38 \cdot 1 \%$; gaps, $35 \cdot 6 \%$ ). Other proteins like the lysozymes LysG from Anser anser (identical, $18 \cdot 0 \%$; similar, $33 \cdot 1 \%$; gaps, $23 \cdot 8 \%$ ) and Gallus gallus LysC are less similar, although they were previously chosen to model the structure of A. tumefaciens VirB1 (Mushegian et al., 1996). Structural superposition shows that despite an almost identical tertiary structure of LysG and Slt70 (Koraimann, 2003), their sequence similarity (identical, $22 \cdot 0 \%$; similar, $36 \cdot 3 \%$; gaps, $32.7 \%$ ) is less than that between Slt70 and VirB1. It was therefore appropriate to use the surface model of Slt70 to visualize regions of VirB1sp interaction with other VirB proteins. If the amino acids identified here by peptide array analysis were important for interactions they would be expected to localize on the surface of a protein. We indeed localized the 
Table 4. Suggested and proven interactions of VirB1

The data for the predicted VirB protein localizations and interactions detected with dihybrid assays are from other publications as indicated and refer to the A. tumefaciens T4SS. IM, inner membrane; OM, outer membrane; ND, Not determined.

\begin{tabular}{|lccccc|}
\hline \multirow{2}{*}{ Protein } & $\begin{array}{c}\text { Localization } \\
\text { (Cascales \& } \\
\text { Christie, 2003) }\end{array}$ & $\begin{array}{c}\text { VirB1 interaction shown by: } \\
\text { (Ward } \text { et } \text { al., 2002) }\end{array}$ & $\begin{array}{c}\text { Pull-down } \\
\text { assay }\end{array}$ & $\begin{array}{c}\text { Co-elution } \\
\text { (Whyld analysis }\end{array}$ & $\begin{array}{c}\text { Peptide } \\
\text { array }\end{array}$ \\
\hline VirB1 & Periplasm & + & ND & + & ND \\
VirB8 & IM & + & ND & - & + \\
VirB9 & OM & + & + & + & + \\
VirB10 & IM & + & - & - & ND \\
VirB11 & IM & + & + & ND & + \\
VirB4 & IM & + & ND & ND & ND \\
\hline
\end{tabular}

binding sites for StrepIIVirB8sp, StrepIIVirB9sp and StrepIIVirB11s on the surface of StrepIIVirB1sp, and these amino acids were not involved in the stabilization of the tertiary structure, which further substantiates the validity of the model. The model suggests that there was no apparent interference with the active-site cleft, but all three proteins bound C-terminally to residues likely to be involved in enzyme activity (regions 2 and 3 boxed in Fig. 5a). Thus, the binding may modulate enzyme activity; this possibility will be directly addressed in future.

Taken together, the results presented here suggest that VirB1s is a self-interacting protein that establishes transient contacts with other VirB proteins, such as VirB8s, VirB9s and VirB11s. A comparison of these results with predictions and results of previous studies is given in Table 4. A large amount of information on the role of VirB1-like proteins was collected in previous studies (Baron et al., 1997; Höppner et al., 2004; Llosa et al., 2000; Mushegian et al., 1996; Ward et al., 2002; Zahrl et al., 2005). Together with this analysis of protein interactions of VirB1s from the $B$. suis T4SS and the results from functional studies conducted with both the A. tumefaciens and B. suis T4SS, the following model was designed describing the function(s) of VirB1. Upon expression of the virB operon, all VirB proteins possessing an $\mathrm{N}$-terminal signal peptide are exported into the periplasm or partially traverse the inner membrane. VirB1 may form a $50 \mathrm{kDa}$ homodimer, which may render the active site inaccessible. The predicted pore size of the peptidoglycan layer permits diffusion of globular proteins smaller than $55 \mathrm{kDa}$, and therefore VirB9, VirB7, VirB5, VirB2 and the VirB1 dimer probably diffuse freely in the periplasm. The contact between VirB1 and VirB9 may be mediated by the C-terminus of VirB1 and lead to activation of the lytic transglycosylase activity of VirB9-bound VirB1. Transient interactions of VirB1 with VirB8 and VirB11 may facilitate this process, which may lead to a conformational change, followed by processing of VirB1 at its VirB1* cleavage site. The enzyme activity may be modulated by binding of VirB8, VirB9 or VirB11 close to active-site residues. Assembly of VirB7 and VirB9, which subsequently recruit other channel components such as VirB10 and VirB8, may accompany the opening of the cell wall. The N-terminal lytic transglycosylase domain of VirB1 (B1N) may subsequently be degraded in order to protect cellular integrity, whereas the $\mathrm{C}$-terminal domain may remain attached to VirB9. VirB1* may exert an additional function in host cell recognition. As VirB1-like proteins can apparently be exchanged between different T4SS (Höppner et al., 2004; Zahrl et al., 2005), the results of these studies will probably be applicable to a wide variety of VirB1-like proteins from T4SS and other secretion systems (Koraimann, 2003).

\section{ACKNOWLEDGEMENTS}

We are indebted to August Böck (Munich, Germany) for continued support and Günter Koraimann (Graz, Austria) for discussions and the communication of results prior to publication. This work was supported by operating grants from the European Union Frame Programme 5 (contract QLK2-CT-2001-01200), by the Canadian Institutes of Health Research (CIHR grant MOP-64300) and by the Canada Foundation for Innovation (CFI) and the Ontario Innovation Trust (OIT) to C. Baron.

\section{REFERENCES}

Atmakuri, K., Ding, Z. \& Christie, P. J. (2003). VirE2, a type IV secretion substrate, interacts with the VirD4 transfer protein at cell poles of Agrobacterium tumefaciens. Mol Microbiol 49, 1699-1713.

Atmakuri, K., Cascales, E. \& Christie, P. J. (2004). Energetic components VirD4, VirB11 and VirB4 mediate early DNA transfer reactions required for bacterial type IV secretion. Mol Microbiol 54, 1199-1211.

Balsinger, S., Ragaz, C., Baron, C. \& Narberhaus, F. (2004). Replicon-specific regulation of small heat shock genes in Agrobacterium tumefaciens. J Bacteriol 186, 6824-6829.

Baron, C., Llosa, M., Zhou, S. \& Zambryski, P. C. (1997). C-terminal processing and cellular localization of VirB1, a component of the Tcomplex transfer machinery of Agrobacterium tumefaciens. J Bacteriol 179, 1203-1210. 
Baumli, S., Hoeppner, S. \& Cramer, P. (2005). A conserved mediator hinge revealed in the structure of the MED7·MED21 (Med7-Srb7) heterodimer. J Biol Chem 280, 18171-18178.

Bayer, M., Eferl, R., Zellnig, G., Terferle, K., Dijkstra, A., Koraimann, G. \& Högenauer, G. (1995). Gene 19 of plasmid R1 is required for both efficient conjugative DNA transfer and bacteriophage R17 infection. J Bacteriol 177, 4279-4288.

Bayer, M., Iberer, R., Bischof, K., Rassi, E., Stabentheiner, E., Zellnig, G. \& Koraimann, G. (2001). Functional and mutational analysis of P19, a DNA transfer protein with muramidase activity. $J$ Bacteriol 183, 3176-3183.

Berger, B. R. \& Christie, P. J. (1994). Genetic complementation analysis of the Agrobacterium tumefaciens virB operon: virB2 through virB11 are essential virulence genes. J Bacteriol 176, 3646-3660.

Bhandari, P. \& Gowrishankar, J. (1997). An Escherichia coli host strain useful for efficient overproduction of cloned gene products with $\mathrm{NaCl}$ as the inducer. J Bacteriol 179, 4403-4406.

Blackburn, N. T. \& Clarke, A. J. (2001). Identification of four families of peptidoglycan lytic transglycosylases. J Mol Evol 52, 78-84.

Burns-Hamuro, L. L., Ma, Y., Kammerer, S. \& 7 other authors (2003). Designing isoform-specific peptide disruptors of protein kinase A localization. Proc Natl Acad Sci U S A 100, 4072-4077.

Cascales, E. \& Christie, P. J. (2003). The versatile bacterial type IV secretion systems. Nat Rev Microbiol 1, 137-149.

Cascales, E. \& Christie, P. J. (2004). Agrobacterium VirB10, an ATP energy sensor required for type IV secretion. Proc Natl Acad Sci U S A 101, 17228-17233.

Celli, J. \& Gorvel, J. P. (2004). Organelle robbery: Brucella interactions with the endoplasmic reticulum. Curr Opin Microbiol 7, 93-97.

Christie, P. J. (2004). Type IV secretion: the Agrobacterium VirB/D4 and related conjugation systems. Biochim Biophys Acta 1694, 219-234.

Comerci, D. J., Martinez-Lorenzo, M. J., Sieira, R., Gorvel, J. P. \& Ugalde, R. A. (2001). Essential role of the VirB machinery in the maturation of the Brucella abortus-containing vacuole. Cell Microbiol 3, 159-168.

Dang, T. A. \& Christie, P. J. (1997). The VirB4 ATPase of Agrobacterium tumefaciens is a cytoplasmic membrane protein exposed at the periplasmic surface. J Bacteriol 179, 453-462.

den Hartigh, A. B., Sun, Y. H., Sondervan, D., Heuvelmans, N., Reinders, M. O., Ficht, T. A. \& Tsolis, R. M. (2004). Differential requirements for VirB1 and VirB2 during Brucella abortus infection. Infect Immun 72, 5143-5149.

Eisenbrandt, R., Kalkum, M., Lai, E. M., Lurz, R., Kado, C. I. \& Lanka, E. (1999). Conjugative pili of IncP plasmids, and the Ti plasmid $\mathrm{T}$ pilus are composed of cyclic subunits. J Biol Chem 274, 22548-22555.

Fullner, K. J. (1998). Role of Agrobacterium virB genes in transfer of $\mathrm{T}$ complexes and RSF1010. J Bacteriol 180, 430-434.

Harlow, E. \& Lane, D. (1988). Antibodies: a Laboratory Manual. Cold Spring Harbor, NY: Cold Spring Harbor Laboratory.

Higgins, D. G. (1994). CLUSTAL V: multiple alignment of DNA and protein sequences. Methods Mol Biol 25, 307-318.

Höltje, J. V. (1998). Growth of the stress-bearing and shapemaintaining murein sacculus of Escherichia coli. Microbiol Mol Biol Rev 62, 181-203.

Hong, P. C., Tsolis, R. M. \& Ficht, T. A. (2000). Identification of genes required for chronic persistence of Brucella abortus in mice. Infect Immun 68, 4102-4107.

Höppner, C., Liu, Z., Domke, N., Binns, A. N. \& Baron, C. (2004). VirB1 orthologs from Brucella suis and pKM101 complement defects of the lytic transglycosylase required for efficient type IV secretion from Agrobacterium tumefaciens. J Bacteriol 186, 1415-1422.

Hwang, H. H. \& Gelvin, S. B. (2004). Plant proteins that interact with VirB2, the Agrobacterium tumefaciens pilin protein, mediate plant transformation. Plant Cell 16, 3148-3167.

Jones, A. L., Shirasu, K. \& Kado, C. I. (1994). The product of the virB4 gene of Agrobacterium tumefaciens promotes accumulation of VirB3 protein. J Bacteriol 176, 5255-5261.

Knoblauch, N. T., Rudiger, S., Schonfeld, H. J., Driessen, A. J., Schneider-Mergener, J. \& Bukau, B. (1999). Substrate specificity of the SecB chaperone. J Biol Chem 274, 34219-34225.

Koraimann, G. (2003). Cell wall degrading enzymes in macromolecular transport systems of Gram-negative bacteria. Cell Mol Life Sci 60, 2371-2388.

Kromayer, M., Wilting, R., Tormay, P. \& Böck, A. (1996). Domain structure of the prokaryotic selenocysteine-specific elongation factor SelB. J Mol Biol 262, 413-420.

Kumar, R. B. \& Das, A. (2001). Functional analysis of the Agrobacterium tumefaciens T-DNA transport pore protein VirB8. J Bacteriol 183, 3636-3641.

Kumar, R. B. \& Das, A. (2002). Polar location and functional domains of the Agrobacterium tumefaciens DNA transfer protein VirD4. Mol Microbiol 43, 1523-1532.

Laemmli, U. K. (1970). Cleavage of structural proteins during the assembly of the head of bacteriophage T4. Nature 227, 680-685.

Lehnherr, H., Hansen, A.-M. \& Ilyina, T. (1998). Penetration of the bacterial cell wall: a family of lytic transglycosylases in bacteriophages and conjugative plasmids. Mol Microbiol 30, 454-457.

Leung, A. K., Duewel, H. S., Honek, J. F. \& Berghuis, A. M. (2001). Crystal structure of the lytic transglycosylase from bacteriophage lambda in complex with hexa- $N$-acetylchitohexaose. Biochemistry 40, 5665-5673.

Liu, J. \& Rost, B. (2003). NORSp: predictions of long regions without regular secondary structure. Nucleic Acids Res 31, 3833-3835.

Livingstone, C. D. \& Barton, G. J. (1993). Protein sequence alignments: a strategy for the hierarchical analysis of residue conservation. Comput Appl Biosci 9, 745-756.

Llanos, R., Chevrier, V., Ronjat, M. \& 7 other authors (1999). Tubulin binding sites on gamma-tubulin: identification and molecular characterization. Biochemistry 38, 15712-15720.

Llosa, M. \& O'Callaghan, D. (2004). Euroconference on the Biology of Type IV Secretion Processes: bacterial gates into the outer world. Mol Microbiol 53, 1-8.

Llosa, M., Zupan, J., Baron, C. \& Zambryski, P. C. (2000). The $\mathrm{N}$ - and C-terminal portions of the Agrobacterium VirB1 protein independently enhance tumorgenesis. J Bacteriol 182, 3437-3445.

Lutzmann, M., Kunze, R., Buerer, A., Aebi, U. \& Hurt, E. (2002). Modular self-assembly of a Y-shaped multiprotein complex from seven nucleoporins. EMBO J 21, 387-397.

Maniatis, T. A., Fritsch, E. F. \& Sambrook, J. (1982). Molecular Cloning: a Laboratory Manual. Cold Spring Harbor, NY: Cold Spring Harbor Laboratory.

Middleton, R., Sjölander, K., Krishnamurthy, N., Foley, J. \& Zambryski, P. (2005). Predicted hexameric structure of the Agrobacterium VirB4 C terminus suggests VirB4 acts as a docking site during type IV secretion. Proc Natl Acad Sci U S A 102, 1685-1690.

Mushegian, A. R., Fullner, K. J., Koonin, E. V. \& Nester, E. W. (1996). A family of lysozyme-like virulence factors in bacterial pathogens. Proc Natl Acad Sci U S A 93, 7321-7326. 
Needleman, S. B. \& Wunsch, C. D. (1970). A general method applicable to the search for similarities in the amino acid sequence of two proteins. J Mol Biol 48, 443-453.

O'Callaghan, D., Cazevieille, C., Allardet-Servent, A., Boschiroli, M. L., Bourg, G., Foulongne, V., Frutos, P., Kulakov, Y. \& Ramuz, M. (1999). A homologue of the Agrobacterium tumefaciens VirB and Bordetella pertussis Ptl type IV secretion systems is essential for intracellular survival of Brucella suis. Mol Microbiol 33, 1210-1220.

Odenbreit, S., Gebert, B., Püls, J., Fischer, W. \& Haas, R. (2001). Interaction of Helicobacter pylori with professional phagocytes: role of the cag pathogenicity island and translocation, phosphorylation and processing of CagA. Cell Microbiol 3, 21-31.

Reimer, U., Reineke, U. \& Schneider-Mergener, J. (2002). Peptide arrays: from macro to micro. Curr Opin Biotechnol 13, 315-320.

Reineke, U., Kramer, A. \& Schneider-Mergener, J. (1999). Antigen sequence- and library-based mapping of linear and discontinuous protein-protein-interaction sites by spot synthesis. Curr Top Microbiol Immunol 243, 23-36.

Rohde, M., Püls, J., Buhrdorf, R., Fischer, W. \& Haas, R. (2003). A novel sheathed surface organelle of the Helicobacter pylori type IV secretion system. Mol Microbiol 49, 219-234.

Rost, B. (1996). PHD: predicting one-dimensional protein structure by profile-based neural networks. Methods Enzymol 266, 525-539.

Schmidt-Eisenlohr, H., Domke, N., Angerer, C., Wanner, G., Zambryski, P. C. \& Baron, C. (1999). Vir proteins stabilize VirB5 and mediate its association with the $\mathrm{T}$ pilus of Agrobacterium tumefaciens. J Bacteriol 181, 7485-7492.

Shamaei-Tousi, A., Cahill, R. \& Frankel, G. (2004). Interaction between protein subunits of the type IV secretion system of Bartonella henselae. J Bacteriol 186, 4796-4801.

Sieira, R., Comerci, D. J., Sanchez, D. O. \& Ugalde, R. A. (2000). A homologue of an operon required for DNA transfer in Agrobacterium is required in Brucella abortus for virulence and intracellular multiplication. J Bacteriol 182, 4849-4855.

Smith, T. F. \& Waterman, M. S. (1981). Overlapping genes and information theory. J Theor Biol 91, 379-380.
Thunnissen, A. M., Dijkstra, A. J., Kalk, K. H., Rozeboom, H. J., Engel, H., Keck, W. \& Dijkstra, B. W. (1994). Doughnut-shaped structure of a bacterial muramidase revealed by X-ray crystallography. Nature 367, 750-753.

van Asselt, E. J., Dijkstra, A. J., Kalk, K. H., Takacs, B., Keck, W. \& Dijkstra, B. W. (1999). Crystal structure of Escherichia coli lytic transglycosylase Slt35 reveals a lysozyme-like catalytic domain with an EF-hand. Structure Fold Des 7, 1167-1180.

van Asselt, E. J., Kalk, K. H. \& Dijkstra, B. W. (2000), Crystallographic studies of the interactions of Escherichia coli lytic transglycosylase Slt35 with peptidoglycan. Biochem 39, 1924-1934.

Ward, D., Draper, O., Zupan, J. R. \& Zambryski, P. C. (2002). Peptide linkage mapping of the A. tumefaciens vir-encoded type IV secretion system reveals novel protein subassemblies. Proc Natl Acad Sci U S A 99, 11493-11500.

Winans, S. C. \& Walker, G. C. (1985). Conjugal transfer system of the $\mathrm{N}$ incompatibility plasmid pKM101. J Bacteriol 161, 402-410.

Yanisch-Perron, C., Vieira, J. \& Messing, J. (1985). Improved M13 phage cloning vectors and host strains: nucleotide sequence of the M13mp18 and pUC18 vectors. Gene 33, 103-119.

Yeo, H.-J. \& Waksman, G. (2004). Unveiling molecular scaffolds of the type IV secretion system. J Bacteriol 186, 1919-1926.

Yeo, H.-J., Yuan, Q., Beck, M. R., Baron, C. \& Waksman, G. (2003). Structural and functional characterization of the VirB5 protein from the type IV secretion system encoded by the conjugative plasmid pKM101. Proc Natl Acad Sci U S A 100, 15947-15962.

Yuan, Q., Carle, A., Gao, C., Sivanesan, D., Aly, K., Höppner, C., Krall, L., Domke, N. \& Baron, C. (2005). Identification of the VirB4VirB8-VirB5-VirB2 pilus assembly sequence of type IV secretion systems. J Biol Chem 280, 26349-26359.

Zahrl, D., Wagner, M., Bischof, K., Bayer, M., Zavecz, B., Beranek, A., Ruckenstuhl, C., Zarfel, G. E. \& Koraimann, G. (2005). Peptidoglycan degradation by specialized lytic transglycosylases associated with type III and type IV secretion systems. Microbiology 151, 3455-3467.

Zupan, J., Muth, T. R., Draper, O. \& Zambryski, P. C. (2000). The transfer of DNA from Agrobacterium tumefaciens into plants: a feast of fundamental insights. Plant $J$ 23, 11-28. 